\title{
Phylogenetic similarity and structure of Agaricomycotina communities across a forested landscape
}

\author{
IVAN P. EDWARDS* and DONALD R. ZAK ${ }^{+}$ \\ ${ }^{*}$ School of Natural Resources E Environment, ${ }^{\dagger}$ Department of Ecology and Evolutionary Biology, University of Michigan, \\ Ann Arbor, MI 48109, USA
}

\begin{abstract}
The Agaricomycotina are a phylogenetically diverse group of fungi that includes both saprotrophic and mycorrhizal species, and that form species - rich communities in forest ecosystems. Most species are infrequently observed, and this hampers assessment of the role that environmental heterogeneity plays in determining local community composition and in driving $\beta$-diversity. We used a combination of phenetic (TRFLP) and phylogenetic approaches [Unifrac and Net Relatedness Index (NRI)] to examine the compositional and phylogenetic similarity of Agaricomycotina communities in forest floor and surface soil of three widely distributed temperate upland forest ecosystems (one, xeric oak - dominated and two, mesic sugar maple dominated). Generally, forest floor and soil communities had similar phylogenetic diversity, but there was little overlap of species or evolutionary lineages between these two horizons. Forest floor communities were dominated by saprotrophic species, and were compositionally and phylogenetically similar in all three ecosystems. Mycorrhizal species represented $30 \%$ to $\mathbf{9 0} \%$ of soil community diversity, and these communities differed compositionally and phylogenetically between ecosystems. Estimates of NRI revealed significant phylogenetic clustering in both the forest floor and soil communities of only the xeric oakdominated forest ecosystem, and may indicate that this ecosystem acts as a habitat filter. Our results suggest that environmental heterogeneity strongly influences the phylogenetic $\beta$-diversity of soil inhabiting Agaricomycotina communities, but has only a small influence on forest floor $\beta$-diversity. Moreover, our results suggest that the strength of community assembly processes, such as habitat filtering, may differ between temperate forest ecosystems.
\end{abstract}

Keywords: Agaricomycetes, Agaricomycotina, ectomycorrhizal, habitat filters, temperate forests, phylogenetic community analysis, saprotrophic

Received 20 July 2009; revision received 30 December 2009; accepted 6 January 2010

\section{Introduction}

Beta diversity is the change in species composition over geographic space (Whittaker 1972). High beta diversity (low community similarity) may result from habitat heterogeneity when species differ in their abilities to colonize distinct habitats (Kerr et al. 2001), and habitat characteristics can therefore be seen as defining aspects of a species $\beta$ - niche (Pickett \& Bazzaz 1978). However,

Correspondence: Ivan P. Edwards, Fax: (734) 936 2195;

E-mail: iedwards@umich.edu high $\beta$-diversity may also be observed if dispersal limitation prevents species from reaching all suitable habitats, or if the nature of the species is such that comprehensive sampling is problematic. Microbial communities may be especially problematic, because high species richness and cryptic growth forms make comprehensive sampling a challenge (Hughes et al. 2001; O'Brien et al. 2005; Lynch \& Thorn 2006). Fungi are an important component of terrestrial ecosystem biodiversity (Hawksworth 2001). Within the fungi, the Agaricomycotina (sensu Hibbett 2006; Agaricomycetes, Dacrymycetes and Tremellomycetes) represent about 
$20 \%$ of all described fungal species (Hawksworth 2001). Saprotrophic Agaricomycotina are important agents of plant litter decomposition (Lynch \& Thorn 2006), whereas ectomycorrhizal species are an important component of the mutualistic symbiotic community beneath Pinus, Picea, Quercus, Populus and Betula (Smith \& Read 1997).

Relatively few studies have considered the role of habitat heterogeneity on fungal $\beta$-diversity, and these have focused on ectomycorrhizal species (Nantel \& Neumann 1992; DeBellis et al. 2006). Plant community composition is considered a primary factor defining the $\beta$-niche of ectomycorrhizal fungi (Molina et al. 1992), because these symbiotic species often exhibit host-specificity (Nantel \& Neumann 1992; Bruns et al. 2002; DeBellis et al. 2006). Edaphic factors also influence ectomycorrhizal species distributions (Nantel \& Neumann 1992; Kranabetter et al. 2009). In comparison with ectomycorrhizal species, much less is understood about the factors affecting saprotrophic species distributions at both the local and landscape scales ( $\mathrm{O}^{\prime}$ Brien et al. 2005; Porter et al. 2008), although edaphic factors may be important here too (Tyler 1992). Saprotrophic Agaricomycotina also rely on plant-derived organic compounds for energy, and because the biochemical composition of litter varies between plant species, the landscape-scale distribution of plant species might also influence the distribution of these species (Tyler 1992; O'Brien et al. 2005; Porter et al. 2008).

At landscape scales (1000s to 100000s of ha), plant community assembly has a strong deterministic component, with local variation in physiographic and edaphic properties strongly affecting species distributions (Barnes et al. 1982; Laliberté et al. 2009). Because of this, landscapes can be conceptualized of as a mosaic of plant communities, each of reasonably predictable composition, and each potentially colonized by a subset of the regional Agaricomycotina species pool (Barnes et al. 1982; Villeneuve et al. 1989; Laliberté et al. 2009).

For communities such as the Agaricomycotina, which are characterized by high species richness and highly variable local species assemblages (Villeneuve et al. 1989; DeBellis et al. 2006; Peay et al. 2007), phylogenetic analyses may provide a better measure of community similarity than more traditional species-based ordination analyses (Lozupone \& Knight 2005; Graham \& Fine 2008). Moreover, the phylogenetic structure of communities might provide insight into dominant community assembly processes (Kembel \& Hubbell 2006; Losos 2008). Yet, the degree to which the Agaricomycotina communities of forest ecosystems are phylogenetically distinct or exhibit significant phylogenetic structure is largely unknown. In this study, we asked three questions: (1) Do the floristic and edaphic characteristics of temperate forest ecosystems significantly affect Agaricomycotina community composition? (2) Are the Agaricomycotina communities of temperate forest ecosystems phylogenetically distinct? (3) What types of phylogenetic structure characterize the Agaricomycotina communities of temperate forest ecosystems? We hypothesized that because of intrinsic floristic and edaphic differences, the Agaricomycotina communities of temperate forest ecosystems should be compositionally distinct. Moreover, we hypothesized that these fungal communities should also be phylogenetically distinct, i.e. that despite local differences in community composition, each different temperate forest ecosystem nevertheless recruits Agaricomycotina from a restricted range of evolutionary lineages. Finally, we hypothesized that the Agaricomycotina communities of distinct forest ecosystems should be phylogenetically clustered. To test these hypotheses, we examined the composition, phylogenetic similarity, and phylogenetic structure of Agaricomycotina communities across a temperate forested landscape, using replicated stands of three common upland forest ecosystem types.

\section{Materials and methods}

\section{Study sites}

Our study was conducted in northwestern Lower Michigan, Lat. $44^{\circ} 48^{\prime}$, Long. $85^{\circ} 48^{\prime}$ (Fig. 1). This region has a temperate climate, with a mean annual temperature of $7.2{ }^{\circ} \mathrm{C}$ and a growing season of 100-150 days. Mean annual precipitation is $81 \mathrm{~cm}$ and precipitation is evenly distributed throughout the year (Albert et al. 1986). We sampled three replicate stands in each of three common and widely distributed upland forest ecosystems: black oak - white oak/Vaccinium (BOWO), sugar maple - red oak/Maianthemum (SMRO), and sugar maple-basswood/Osmorhiza (SMBW) ecosystems; they are named for the dominant overstory tree species and a characteristic ground flora species (Host

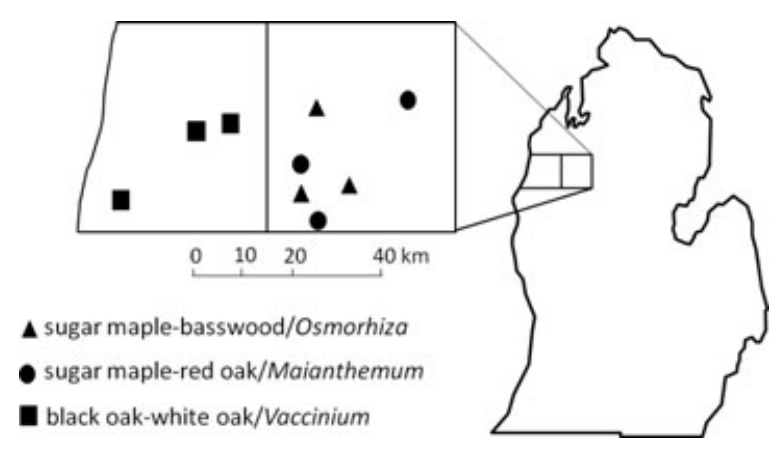

Fig. 1 A map of Lower Michigan showing the location of nine replicate stands in three upland forest ecosystems. 
AGARICOMYCOTINA COMMUNITY PHYLOGENETICS 1471

Table 1 Soil and vegetation characteristics of three upland forest ecosystems in northern Lower Michigan, USA

\begin{tabular}{|c|c|c|c|}
\hline & \multicolumn{3}{|l|}{ Ecosystem type } \\
\hline & Black oak/white oak & Sugar maple/red oak & Sugar maple/Basswood \\
\hline & BOWO & SMRO & SMBW \\
\hline Overstory species & $\begin{array}{l}\text { Quercus velutina* } \\
\text { Quercus alba } \\
\text { Quercus rubra } \\
\text { Acer rubrum } \\
\text { Populus grandidentata }\end{array}$ & $\begin{array}{l}\text { Q. rubra } \\
\text { A. rubrum } \\
\text { P. grandidentata } \\
\text { Fagus grandifolia } \\
\text { O. virginiana } \\
\text { Tilia americana } \\
\text { Acer saccharum* }\end{array}$ & $\begin{array}{l}\text { A. saccharum* } \\
\text { Tilia americana } \\
\text { P. grandidentata } \\
\text { F. grandifolia } \\
\text { Fagus americana } \\
\text { O. virginiana }\end{array}$ \\
\hline Ground flora & Vaccinium & Maianthenum & Ozmorhiza \\
\hline Litter Lignin $(\mathrm{mg} / \mathrm{g}) \dagger$ & 340 & 300 & 260 \\
\hline Litter Cellulose $(\mathrm{mg} / \mathrm{g}) \dagger$ & 440 & 440 & 500 \\
\hline Soil Type & Entic Haplorthod & Typic Haplorthod & Typic Haplorthod \\
\hline $\mathrm{pH} \ddagger$ & 3.9 & 4.1 & 5.5 \\
\hline Organic C $(\mathrm{g} / \mathrm{kg}) \ddagger$ & 440 & 390 & 550 \\
\hline Total $\mathrm{N} \ddagger\left(\mu \mathrm{g} / \mathrm{N} \mathrm{g}^{-1}\right)$ & 1913 & 1835 & 3040 \\
\hline Net $\mathrm{N}$ mineralization $\ddagger\left(\mu \mathrm{g} \mathrm{N} \mathrm{g}^{-1}\right.$ year $^{-1}$ ) & 313 & 382 & 426 \\
\hline Net Nitrification $\ddagger\left(\mu \mathrm{g} \mathrm{N} \mathrm{g}{ }^{-1}\right.$ year $^{-1}$ ) & 18 & 43 & 364 \\
\hline
\end{tabular}

*Indicates canopy dominant.

+Litter characteristics from Blackwood et al. 2007.

$\ddagger$ All soil properties measured in 0-3.8 cm depth, from Zak et al. 1990.

et al. 1988; Zak et al. 1990; Table 1). Distance between replicate stands of an ecosystem type ranged from $0.5-35 \mathrm{~km}$ (average, $12.9 \mathrm{~km}$ ); all stands are late successional, with overstory trees that are approximately 100 years old (Table 1 ).

\section{Field sampling}

Because ectomycorrhizal and saprotrophic species are often vertically stratified between forest floor and soil (Lindahl et al. 2007), we sampled these two horizons separately. Within each stand, 12 forest floor and 12 surface soil samples were collected at 5-m intervals along two parallel transects spanning a pre-existing $30-\mathrm{m} \times 10-\mathrm{m}$ plot. At each sampling location, forest floor was collected from a $0.1 \mathrm{~m}^{2}$ area and surface soil was collected to a depth of $5 \mathrm{~cm}$ with a $2.5 \mathrm{~cm}$ diameter soil corer. In the SMBW ecosystem, forest floor $\left(\mathrm{O}_{\mathrm{i}}\right)$ abruptly gives way to the underlying mineral soil, whereas in the BOWO and SMRO ecosystems, a 3 to 5 -mm thick $\mathrm{O}_{e}$ horizon forms on the surface of the mineral soil. This horizon, which is densely interpenetrated by fine roots, is continuous in the BOWO ecosystem and discontinuous in the SMRO ecosystem. We collected the $\mathrm{O}_{e}$ as part of the surface soil, not the forest floor. Forest floor and surface soil samples collected in each plot were combined to produce one composite forest floor and one composite surface soil sample for each replicate stand; these samples were stored on ice prior to DNA extraction.

\section{Sample preparation and DNA extraction}

We used a soil-washing technique prior to DNA extraction in order to increase the probability of sampling DNA from actively growing hyphae, rather than potentially dormant basidiospores (Bååth 1988; Lynch \& Thorn 2006). Briefly, soil samples were homogenized by hand and passed through a $2-\mathrm{mm}$ sieve to remove roots and coarse fragments. Ten grams of sieved, homogenized soil was shaken with $100 \mathrm{~mL}$ of $0.1 \mathrm{~m}$ sodium pyrophosphate for $1 \mathrm{~h}$, and the slurry washed through sieves of $0.25 \mathrm{~mm}$ and $0.053 \mathrm{~mm}$ mesh with $\sim 4 \mathrm{~L}$ of deionized water (Lynch \& Thorn 2006). DNA was extracted from the $0.053-0.25 \mathrm{~mm}$ fraction using PowerMax TM Soil kits (Mo Bio) as per manufacturer's instructions. Forest floor samples were chopped (Hamilton Beach R 10 speed blender) to facilitate homogenization and subsequently treated as described for soils, except that $2.5 \mathrm{~g}$ of material was washed and used for DNA extraction. Duplicate DNA extractions were performed on each sample in order to assess the effectiveness of the homogenization procedure; DNA was stored at $-80{ }^{\circ} \mathrm{C}$. 


\section{Polymerase chain reaction}

The entire ribosomal rDNA internal transcribed spacer region and approximately 400 bases of the large-subunit rDNA was selectively amplified from DNA extracts using primers ITS1F (5'-CTT GGT CAT TTA GAG GAA GTA A-3', Gardes \& Bruns 1993) and LR21 (5'-ACT TCA AGC GTT TCC CTT T-3', Hopple \& Vilgalys 1994). ITS1F is considered a general fungal primer and was designed to minimize co-amplification of non-fungal DNA, while LR21 preferentially amplifies basidiomycete DNA. We used the same primers for both cloning and Terminal Restriction Fragment Length Polymorphism (TRFLP), with the ITS1F primer labelled with 6-FAM for TRFLP. Each PCR reaction cocktail contained 50-200 ng of soil DNA, $200 \mathrm{~nm}$ dNTPs, 1X PCR buffer including $1.5 \mathrm{~mm} \mathrm{MgCl}_{2}$ (Roche), $0.5 \mu \mathrm{m}$ of each primer and $50 \mu \mathrm{g}$ of BSA. After an initial denaturation step of $3 \mathrm{~min}$ at $94^{\circ} \mathrm{C}, 35$ cycles (TRFLP) or 25 cycles (clone libraries) of $94^{\circ} \mathrm{C}$ for $30 \mathrm{~s}$, $55{ }^{\circ} \mathrm{C}$ for $45 \mathrm{~s}$ and $72{ }^{\circ} \mathrm{C}$ for $90 \mathrm{~s}$ were carried out using Stratagene PCR cyclers (La Jolla, CA). A final extension step of $72{ }^{\circ} \mathrm{C}$ for $15 \mathrm{~min}$ was used to minimize the production of pseudo-restriction products (Egert \& Friedrich 2003).

\section{Cloning}

Triplicate PCR products from each horizon of the three replicate stands in each ecosystem $(n=3)$ were combined and purified (UltraClean PCR Clean-up, Mo Bio) prior to cloning into $\mathrm{pCR}^{\circledR}$ 2.1-TOPO ${ }^{\circledR}$ using the TOPO TA Cloning kit (Invitrogen). Thirty-two clones for each soil horizon in each stand were selected to create six 96-clone libraries, one for each horizon $(n=2)$ of each ecosystem $(n=3)$. Clones were grown overnight in Luria broth supplemented with $10 \%$ glycerol, and sent to the University of Georgia for bidirectional sequencing with M13F and M13R primers. Sequence quality was assessed by visual inspection, and full length contiguous sequences for each clone were constructed in Geneious 3.7.0 (Biomatters Ltd.).

\section{Terminal restriction fragment length polymorphism}

For TRFLP, approximately $500 \mathrm{ng}$ of purified PCR product (see above) was digested overnight with $10 \mathrm{U}$ of HaeIII (Promega) at $37^{\circ} \mathrm{C}$. Digests were desalted with Microcon YM-30 centrifugal filters (Millipore) prior to genotyping. All samples were mixed with ROX 1000 size marker, and genotyping was performed at the University of Michigan's Core Sequencing Facility using an ABI 3730XL DNA Sequencer with a 96 capillary array. Terminal restriction fragment lengths were determined relative to the ROX 1000 size standard with Genemarker 1.51 (SoftGenetics). Each digest was run four times, (duplicates on two separate runs) and all TRF greater than 50 fluorescence units that occurred in at least both duplicate electropherograms of each run were scored in a presence-absence matrix. Initial sensitivity analyses demonstrated that the number of TRF peaks observed in each sample was essentially independent of total signal intensity after a threshold intensity of 20000 fluorescence units was reached; samples were reanalyzed if they did not meet this minimum intensity. Terminal restriction fragment length profiles obtained from each sample were highly reproducible, and we estimated measurement precision as $\pm 0.5 \mathrm{bp}$ for TRF up to $1000 \mathrm{bp}$, and $\pm 1.0 \mathrm{bp}$ for TRF $1000-1200 \mathrm{bp}$.

\section{Operational taxonomic units}

There is no ideal single-gene approach to defining either fungal species or the phylogeny of the Agaricomycotina (Taylor et al. 2000; Hibbett 2006; Nilsson et al. 2008). Generally, the rDNA ITS has a higher degree of resolution at the subgeneric level than more conserved regions such as rDNA 28S (Nilsson et al. 2008). However, alignment problems beyond the generic or family level due to the variability of the non-coding ITS1 and ITS2 spacers make this region of little use in phylogenetic community analysis. Some recent studies have defined fungal operational taxonomic units (OTUs) in terms of rDNA $28 \mathrm{~S}$ sequence similarity, using $99 \%$ sequence similarity as an arbitrary cutoff (Lynch \& Thorn 2006; Porter et al. 2008). While pragmatic, this approach is also conservative and may unintentionally lump together closely related species (Lynch \& Thorn 2006). In this study, we used a twostep procedure to define OTUs in order to capitalize on the information provided by both ITS and rDNA 285 regions recovered with the ITS1F-LR21 primer set. First, we created an rDNA 285 alignment of all sequences using Clustal W (Thompson et al. 1997) within Geneious (Drummond et al. 2008). This alignment was used to generate a similarity matrix in MEGA 4.0 (Tamura et al. 2007), and subsequently to cluster sequences into groups based on $\geq 99 \%$ similarity using the furthest neighbour algorithm in DOTUR (Schloss \& Handelsman 2005). Subsequently, for each of the rDNA $28 \mathrm{~S}$ groups that included more than one sequence, we repeated this procedure using full length (ITS $+28 \mathrm{2}$ ) alignments and defined OTUs at $\geq 97 \%$ similarity. Sequences representing each OTU have been deposited in GenBank (GU328501-GU328639). Non-parametric estimates of species richness (Chao I and Jacknife) were also calculated by DOTUR (Fig. S1 Supporting information). 


\section{OTU identification and chimera checking}

BLAST searches were performed for all OTUs to retrieve the top-matching sequence from GenBank. To check for chimeric sequences, we repeated the BLAST searches using first the ITS sequence and then the $28 \mathrm{~S}$ fragment. When the two searches returned dramatically different results, we considered this as possible evidence of a chimera and the sequence was excluded from subsequent analyses. After examination of preliminary phylogenetic trees with the reference sequences, only OTUs which placed clearly in the Agaricomycota were retained for analysis.

\section{Phylogenetics}

The rDNA $28 \mathrm{~S}$ gene fragments $(\sim 450 \mathrm{bp})$ from each OTU were aligned using Clustal W (Thompson et al. 1997) in Geneious (Drummond et al. 2008), and the alignment was manually edited. The hypervariable D1 region was excluded from the alignment. ModelTest (Posada \& Crandall 1998) was used to compare evolutionary models, and a bootstrapped neighbour-joining tree created using MEGA 4.0. Ascomycete sequences were used to root the tree.

\section{Gradient analysis}

We used Detrended Correspondence Analysis (DCA) to assess the similarity of the Agaricomycotina communities in each horizon across the landscape, and Canonical Correspondence Analysis (CCA, Ter Braak 1986) to determine the significance of the species - environment correlation. Species data consisted of a HaeIII TRFLP presence-absence matrix, and we assumed that each unique TRF peak represented a single species. This assumption is known to not be strictly true (Edwards \& Turco 2005; Avis et al. 2006; Dickie \& FitzJohn 2007) but it is unavoidable in the absence of extensive a priori knowledge of these communities. Environmental parameters included in CCA were: relative abundance of overstory species, soil $\mathrm{pH}$, soil $\mathrm{N}$ mineralization rate $\left(\mathrm{N}_{\text {min }}\right)$, total organic carbon (TOC), and forest floor cellulose and lignin contents (Zak et al. 1990; Blackwood et al. 2007). Significance of community-environment correlations was tested with a Monte Carlo test, using 999 unrestricted permutations. In both DCA and CCA, forest floor and soil fingerprints were examined together, and singleton TRFs were not removed, although their influence on the ordination was minimized using the 'downweight rare species' option in CANOCO (Biometris, Wageningen). TRFLP fingerprints were also used to assess the influence of distance between sites on fungal community similarity. Finger- print similarity was estimated as Sorensen's index, ( 1 = identical, $0=$ no overlap) and this was regressed against distance $(\mathrm{km})$ between study sites using linear regression. Regressions for litter and soil horizons were performed separately using SAS 8.1.

\section{Phylogenetic similarity, diversity and structure}

The phylogenetic similarity of the Agaricomycotina communities was assessed using Unifrac (Lozupone \& Knight 2005). UniFrac estimates the distance between communities as the fraction of the branch length of the phylogenetic tree that leads to descendants from either one environment or another, but not both; we used this distance matrix to cluster environments using Jackknifed UPGMA. Phylocom-3.40 (Webb 2000) was used to calculate Faith's index of phylogenetic diversity (PD, Faith 1992) and the Net Relatedness Index (NRI). PD is defined as the minimum branch length spanning any given set of species on a phylogenetic tree. The phylogenetic structure of each community was estimated with the Net Relatedness Index (NRI, Webb 2000). NRI is defined as $\left[-\left(\mathrm{MPD}-\mathrm{MPD}_{\text {null }}\right) / \mathrm{SD}\left(\mathrm{MPD}_{\text {null }}\right)\right]$, where MPD is the mean pairwise phylogenetic distance between species in a community, MPD $\mathrm{Mull}_{\text {is }}$ the mean MPD for 1000 random communities, and $S D\left(M P D_{\text {null }}\right)$ is the standard deviation. The significance of phylogenetic pattern was determined relative to 999 randomly assembled communities created under Phylocom's null model 2 (Webb et al. 2002) whereby species richness was maintained in each sample, and species were drawn randomly from the list of all species present in all samples. Because the significance of phylogenetic structure can be sensitive to tree topology (Swenson 2009), we manually edited the basic input tree to collapse all unsupported nodes and repeated the analysis with the resulting polytomy tree.

\section{Results}

\section{Gradient analysis}

Overall, we recorded 171 unique 5' HaeIII terminal restriction fragments (TRF) ranging in size from $73 \pm 0.5$ to $1062 \pm 1$ base pairs; 66 of these were unique to the forest floor, 86 were unique to surface soil and 19 were recovered from both horizons. Most TRF were recovered from a single stand, and $\sim 90 \%$ were recovered from three stands or less (Supporting information, Fig. S2). Sorensen similarity values between sites ranged from 0.08 to 0.74 in the forest floor and from 0.0 to 0.46 in the soil, and similarity was independent of distance between sites (soil, $P=0.68$; forest floor $P=0.85)$. 


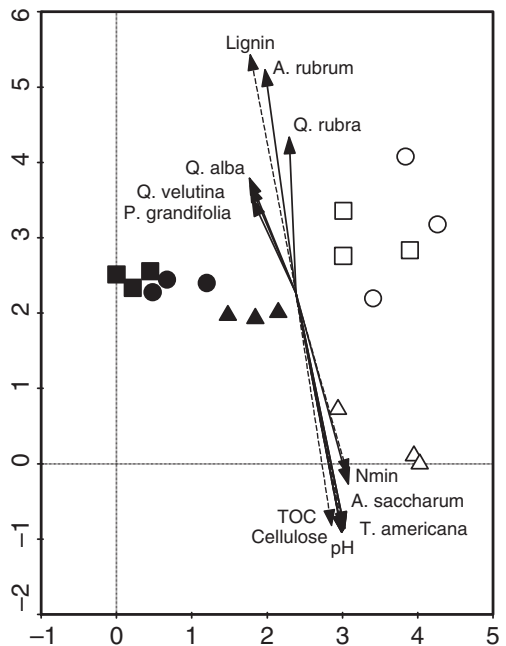

Fig. 2 Detrended Correspondence Analysis (DCA) biplot displaying the similarity of nine upland hardwood forest stands in terms of fungal community composition. Soil (open symbols) and forest floor (closed symbols) are presented separately. The nine points represent three replicate stands in three contrasting upland forest types; squares: black oak/white oak; circles: sugar maple - red oak; triangles: sugar maple - basswood). Soil properties (dashed vectors), and overstory plant abundances (solid vectors) are plotted as passive vectors. Axes scale, standard deviation. The first correspondence axis explains $20.6 \%$ of the variance, and the second axis $8.8 \%$. Both axes are highly significant $(P=0.002)$.

Detrended Correspondence Analysis separated forest floor from soil TRFLP profiles along a primary axis with a gradient length of $4.3 \mathrm{SD}$ that accounted for $12.7 \%$ of the overall variance (Fig. 2). A second axis with a gradient length of $4.1 \mathrm{SD}$, accounted for an additional $7.9 \%$ of the variance. This second axis provided little extra separation within the forest floor communities, but tended to separate the oak soil horizon fungal communities from the sugar maple-basswood soil horizon communities. The primary CCA axis accounted for $12.0 \%$ of the variance and was significantly correlated to soil horizon $\left(r^{2}=0.98, P=0.01\right)$. The second canonical axis was strongly correlated to Quercus and Acer distribution, net $\mathrm{N}$ mineralization rate, and forest floor lignin and cellulose contents. This second axis accounted for a further $6.6 \%$ of the variance and was also significant $(P=0.02)$.

\section{Phylogenetic diversity}

We obtained non-chimeric rDNA ITS-28S sequences from 529 clones, and initial BLAST and phylogenetic analyses indicated that the majority of these were fungal and that 461 placed in the Agaricomycotina. Clustering of these sequences based on 99\% rDNA $28 \mathrm{~S}$ similarity produced 106 groups, 44 of which included multiple sequences. Full length rDNA ITS-28S alignments within each of these groups showed sequence similarity levels of $87-100 \%$ (average $96.03 \%$ ), and based on $97 \%$ full length similarity, we recovered 139 OTU with rDNA $28 \mathrm{~S}$ similarities of $99-100 \%$ (Table S1, Supplementary material). Based on the rDNA $28 \mathrm{~S}$ alignment, Chao I and Jackknife estimates of species richness ranged from 170 to 295, and the curves for the overall library had positive slopes, suggesting that further sampling would likely have recovered more OTUs (Fig. S1, Supporting information).

The phylogenetic dataset included 141 rDNA 285 sequences (including two Ascomycete outgroup tax) aligned in 468 positions. Forty ambiguous sites were excluded from the analysis, and of the remaining 428, 192 were variable and 236 constant. ModelTest indicated that a Kimura 2-parameter model with a gamma function provided an efficient fit to the data, and we used this to create a bootstrapped neighbour-joining tree (Supporting information, Fig. S3). Generally, terminal groups in the phylogeny were moderately to well supported, but deeper nodes were not (Supporting information, Fig. S3). Despite this, the overall topology of the tree accorded reasonably well with recent analyses of the Agaricomycota (e.g. Hibbett 2006). Both Agaricomycetes and Tremellomycetes were recovered (Supporting information Fig. S3) and the OTUs represented species from at least 15 orders of Agaricomycotina (Table 2). Agaricomycetes represented $64-96 \%$ of the OTUs recovered from each forest ecosystem, and Agaricomycete OTU richness was higher in soil than forest floor (Table 2). Tremellomycetes represented $6-36 \%$ of OTUs within each forest ecosystem, and Tremellomycete OTU richness was higher in the forest floor (Table 2).

Within the Agaricomycetes, Agaricales were dominant and were significantly more diverse in soil than forest floor (Table 2). The diversity of Russulales and Boletales also tended to be greater in soil than forest floor (Table 2). In contrast, Cantharellales were exclusively recovered from forest floor (Table 2). In both forest floor and soil, phylogenetic diversity was lowest in BOWO ecosystem and highest in SMBW ecosystem (Table 3). Although we recovered more OTUs from the soil than the forest floor, species in the forest floor tended to encompass a greater phylogenetic diversity both at the landscape scale and within each of the three forest ecosystems (Table 3).

\section{Community phylogenetic similarity}

There was little overlap in community membership between forest floor and soil, with only two OTUs recovered from both. Moreover, as for TRFLP, most 
Table 2. Comparison of the Agaricomycotina communities of three upland forest ecosystems. Data is expressed as proportion of community OTU richness

\begin{tabular}{|c|c|c|c|c|c|c|c|}
\hline \multirow[b]{2}{*}{ Order } & \multicolumn{3}{|c|}{ Forest floor } & \multicolumn{3}{|l|}{ Soil } & \multirow[b]{2}{*}{ Comment } \\
\hline & BOWO & SMRO & SMBW & BOWO & SMRO & SMBW & \\
\hline Agaricomycetes & 0.74 & 0.64 & 0.66 & 0.96 & 0.96 & 0.94 & \\
\hline Agaricales & 0.39 & 0.39 & 0.28 & 0.52 & 0.67 & 0.44 & Greater richness in soil \\
\hline Cantharellales & 0.17 & 0.11 & 0.19 & 0.00 & 0.00 & 0.00 & Forest floor only \\
\hline Other* & 0.09 & 0.04 & 0.03 & 0.00 & 0.07 & 0.06 & \\
\hline Russullales & 0.04 & 0.04 & 0.03 & 0.24 & 0.07 & 0.09 & Greater richness in soil \\
\hline Stereales & 0.04 & 0.00 & 0.00 & 0.00 & 0.00 & 0.00 & \\
\hline Atheliales & 0.00 & 0.00 & 0.00 & 0.12 & 0.00 & 0.00 & BOWO soil only \\
\hline Boletales & 0.00 & 0.00 & 0.03 & 0.08 & 0.00 & 0.15 & \\
\hline Geastrales & 0.00 & 0.00 & 0.00 & 0.00 & 0.00 & 0.03 & \\
\hline Phallales & 0.00 & 0.00 & 0.03 & 0.00 & 0.00 & 0.00 & \\
\hline Polyporales & 0.00 & 0.00 & 0.00 & 0.00 & 0.00 & 0.03 & \\
\hline Sebacinales & 0.00 & 0.00 & 0.03 & 0.00 & 0.00 & 0.12 & SMBW only \\
\hline Thelephorales & 0.00 & 0.00 & 0.03 & 0.00 & 0.07 & 0.00 & \\
\hline Trechisporales & 0.00 & 0.07 & 0.00 & 0.00 & 0.07 & 0.06 & \\
\hline Tremellomycetes & 0.26 & 0.36 & 0.34 & 0.00 & 0.04 & 0.06 & \\
\hline Filobasidiales & 0.17 & 0.18 & 0.28 & 0.00 & 0.04 & 0.03 & Greater richness in forest floor \\
\hline Tremellales & 0.04 & 0.14 & 0.06 & 0.00 & 0.00 & 0.03 & Greater richness in forest floor \\
\hline Cystofilobasidiales & 0.04 & 0.04 & 0.00 & 0.00 & 0.00 & 0.00 & Greater richness in forest floor \\
\hline
\end{tabular}

*Other includes OTUs that placed in poorly represented groups such as Corticiales and Gomphales.

OTUs showed a very limited distribution across the landscape, with $\sim 97 \%$ of all OTU recovered from three or less stands (Fig. S2 Supporting information). Only three OTUs were recovered from all three ecosystems and all of these were recovered from the forest floor. OTUs that placed within known ectomycorrhizal clades were recovered almost exclusively from the soil horizon. Putative ectomycorrhizal OTUs dominated the soil in the BOWO ecosystem, in which they comprised $92 \%$ of all OTUs; however, these organisms were less prominent in SMRO and SMBW ecosystems (30\% and 25\% respectively, Fig. S4 Supporting information). No ectomycorrhizal OTU was recovered from all three ecosystems, although some showed high fidelity to a particular forest type (e.g. Piloderma sp. and some Russula spp. in BOWO soils).

UniFrac unambiguously distinguished soil and forest floor communities (UniFrac $P<0.01$ ). Within the forest floor, UniFrac revealed a higher level of similarity between BOWO and SMRO fungal communities than between these and SMBW (Fig. 3). Despite this, the UniFrac metric indicated no significant difference between these three communities in terms of Agaricomycotina lineages (BOWO vs. SMRO, $P=0.26$; BOWO vs. SMBW, $P=0.20$; $\mathrm{SMRO}$ vs. SMBW $P=0.26$ ). Within the soil horizon, UniFrac revealed a higher level of similarity between SMBW and SMRO fungal communities than between these and BOWO (Fig. 3). Moreover, within the soil horizon the UniFrac metric indicated significant differences between the BOWO fungal community and those of SMRO and SMBW (BOWO vs. SMRO, $P=0.03$; BOWO vs. SMBW, $P=0.02$ ). There was also a marginally significant difference between the SMRO soil and SMBW soil communities $(P=0.08)$.

\section{Community phylogenetic structure}

Forest floor and soil horizon Agaricomycotina communities had similar phylogenetic diversity when pooled across the three ecosystems, and moreover both communities had positive NRI values, suggesting phylogenetic clustering; however this was not statistically significant (Table 3).

Both NRI and PD values were highly variable at the stand scale $\left(300 \mathrm{~m}^{2}\right)$ and no clear trends were apparent (Fig. 4). Twelve of the 18 communities had positive NRI values; although for only three of these, two BOWO soil horizon and one BOWO forest floor, did these values significantly exceed null expectations. The remaining six communities had negative NRI values, suggesting phylogenetic overdispersion, but this was only significant in one SMBW forest floor community.

We estimated the effect of spatial scale by pooling replicate stand data. At this scale $\left(900 \mathrm{~m}^{2}\right)$ the Agaricomycotina soil horizon community of the BOWO ecosystem exhibited significant phylogenetic clustering, while the forest floor community of SMBW was 
Table 3. Phylogenetic diversity and structure in the Agaricomycotina communities associated with three upland forest ecosystems in northern Lower Michigan, USA

\begin{tabular}{|c|c|c|c|c|c|c|c|}
\hline Ecosystem & Horizon & $N^{*}$ & P.D.† & NRI & Obs $>\operatorname{Sim} \ddagger$ & $P \S$ & Pattern \\
\hline \multicolumn{8}{|c|}{ Agaricomycotina } \\
\hline ALL & Forest floor & 72 & 0.569 & 0.5608 & 699 & n.s. & Random \\
\hline ALL & Soil & 75 & 0.566 & 1.2100 & 892 & 0.11 & Random \\
\hline \multicolumn{8}{|c|}{ Agaricomycotina } \\
\hline BOWO & Forest floor & 23 & 0.231 & 0.8582 & 813 & n.s. & Random \\
\hline SMRO & Forest floor & 28 & 0.278 & -0.0629 & 479 & n.s. & Random \\
\hline SMBW & Forest floor & 32 & 0.353 & -1.1688 & 120 & 0.12 & Random \\
\hline BOWO & Soil & 25 & 0.182 & 2.4011 & 988 & 0.01 & Clustered \\
\hline SMRO & Soil & 27 & 0.304 & -0.5923 & 289 & n.s. & Random \\
\hline SMBW & Soil & 34 & 0.344 & 0.1311 & 544 & n.s. & Random \\
\hline \multicolumn{8}{|c|}{ Agaricomycotina (polytomy) } \\
\hline BOWO & Forest floor & 23 & 0.205 & 1.0359 & 856 & n.s. & Random \\
\hline SMRO & Forest floor & 28 & 0.265 & -0.3169 & 362 & n.s. & Random \\
\hline SMBW & Forest floor & 32 & 0.329 & -1.0367 & 142 & 0.14 & Random \\
\hline BOWO & Soil & 25 & 0.157 & 2.4724 & 993 & 0.006 & Clustered \\
\hline SMRO & Soil & 27 & 0.267 & -0.0179 & 488 & n.s. & Random \\
\hline SMBW & Soil & 34 & 0.307 & 0.5834 & 724 & n.s. & Random \\
\hline \multicolumn{8}{|c|}{ Agaricomycetes } \\
\hline BOWO & Forest floor & 17 & 0.150 & 1.9247 & 969 & 0.03 & Clustered \\
\hline SMRO & Forest floor & 18 & 0.173 & 0.8493 & 795 & n.s. & Random \\
\hline SMBW & Forest floor & 21 & 0.252 & -0.0310 & 469 & n.s. & Random \\
\hline BOWO & Soil & 25 & 0.182 & 2.4657 & 991 & 0.01 & Clustered \\
\hline SMRO & Soil & 26 & 0.287 & -0.5648 & 292 & n.s. & Random \\
\hline SMBW & Soil & 32 & 0.301 & 0.4881 & 698 & n.s. & Random \\
\hline \multicolumn{8}{|c|}{ Agaricomycetes (polytomy) } \\
\hline BOWO & Forest floor & 17 & 0.124 & 1.9421 & 975 & 0.02 & Clustered \\
\hline SMRO & Forest floor & 18 & 0.155 & 0.5466 & 688 & n.s. & Random \\
\hline SMBW & Forest floor & 21 & 0.227 & -0.1463 & 441 & n.s. & Random \\
\hline BOWO & Soil & 25 & 0.157 & 2.4060 & 988 & 0.01 & Clustered \\
\hline SMRO & Soil & 26 & 0.251 & 0.0911 & 529 & n.s. & Random \\
\hline SMBW & Soil & 32 & 0.265 & 0.9456 & 819 & n.s. & Random \\
\hline
\end{tabular}

${ }^{*} N$, number of OTU.

tFaith's index of phylogenetic diversity.

$\ddagger$ Number of times that NRI values of the natural community exceeded those of 999 random simulated communities generated under Phylocom null model 2.

$\S P$-value for a one-sided test.

overdispersed, although this was not significant (Table 3). We also estimated the effects of taxonomic scale by restricting the analysis to the Agaricomycetes within the pooled $900 \mathrm{~m}^{2}$ communities; significant clustering was still observed in the BOWO soil community, but in addition the BOWO forest floor Agaricomycete community exhibited significant phylogenetic clustering (Table 3). In contrast, with Tremellomycetes removed from the analysis, the SMBW community no longer exhibited overdispersion (Table 3). Finally, we examined the robustness of these results to topological inaccuracy in the phylogenetic tree; all unsupported branches were collapsed, and NRIs recalculated for Agaricomycotina and Agaricomycetes at the $900 \mathrm{~m}^{2}$ scale using the resultant poorly resolved polytomy.
Incomplete basal resolution had no effect on the phylogenetic patterns observed (Table 3).

\section{Discussion}

Do the floristic and edaphic characteristics of temperate forest ecosystems significantly affect Agaricomycotina community composition?

Generally, there was a greater similarity between the TRFLP community fingerprints of replicate stands of each forest ecosystem type than there was between fingerprints of differing ecosystem types (Fig. 2). Furthermore, constrained (CCA) analysis revealed significant correlations between fingerprint similarity and 


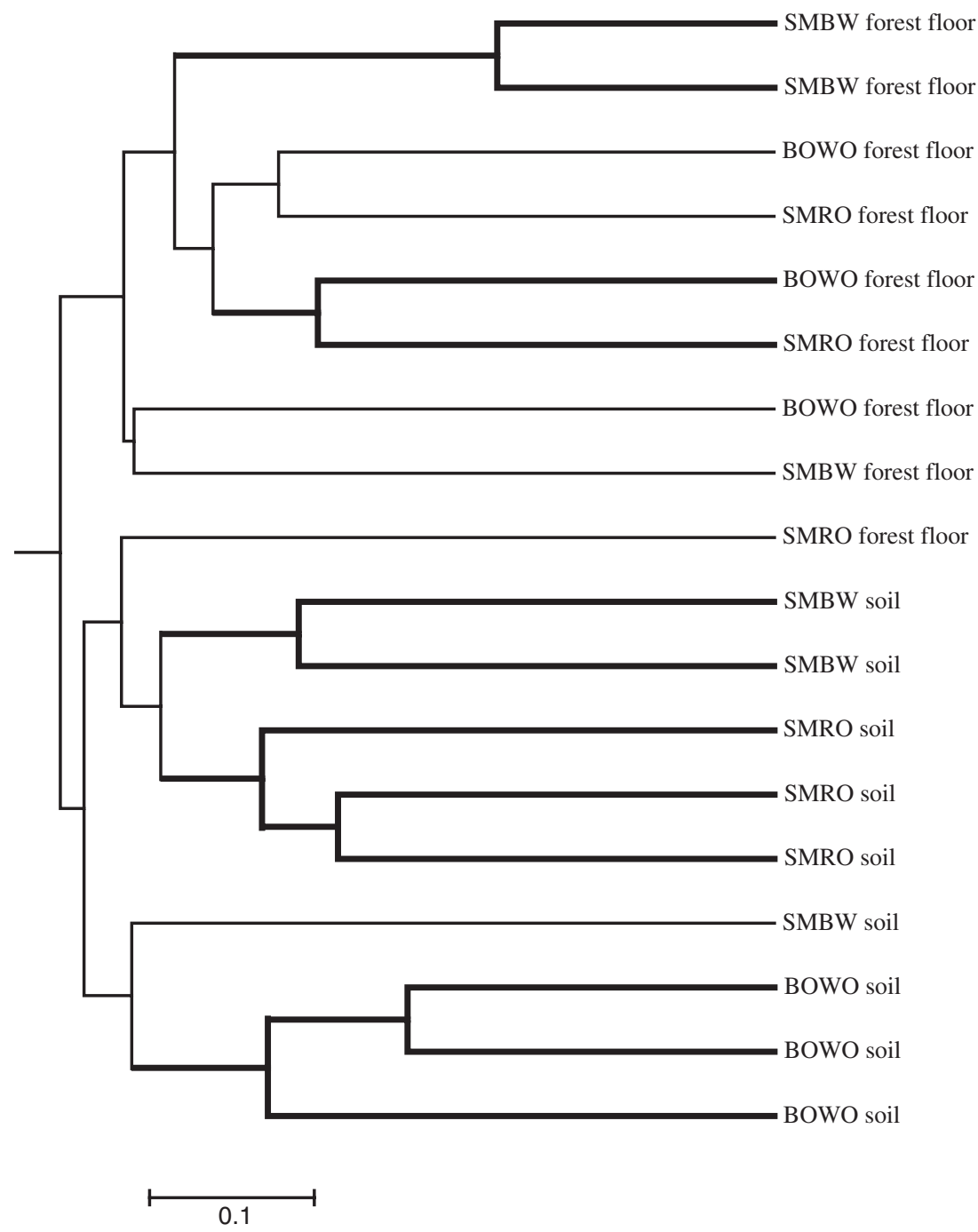

Fig. 3 Phylogenetic similarity of the basidiomycete communities from forest floor and soil horizons of three replicate stands in three contrasting upland hardwood forest ecosystems on UPGMA clustering of the Unifrac distance matrix. BOWO: black oak/white oak; SMRO: sugar maple - red oak; SMBW: sugar maple - basswood. Bold branches and nodes are supported by $>70 \%$ of Jackknife replicates.

environmental factors such as soil horizon and the floristic and edaphic properties of the three upland forest ecosystems. As such, fingerprinting suggested that heterogeneity of the forested landscape increases $\beta$-diversity, because some degree of niche differentiation affects the distributions of Agaricomycotina species. These results are consistent with previous gradient analyses of the ectomycorrhizal community - environment relationship, despite differences in methodology (Nantel \& Neumann 1992; DeBellis et al. 2006). Yet, the relationship between community composition and forest ecosystem characteristics accounted for only a small proportion $(<10 \%)$ of the variance in species distributions. Conceivably, methodological limitations of the TRFLP approach might mask the true strength of the relationships between species distributions and forest ecosystem characteristics (Dickie \& FitzJohn 2007). In this study, we employed TRFLP in what has been termed a 'peak - profiling TRFLP' approach (Dickie \& FitzJohn 2007). The effec- tiveness of peak-profiling depends on primer selectivity and on the assumption that each unique TRF peak represents a distinct species (Dickie \& FitzJohn 2007). The ITS1F-LR21 primer pair is not selective for Agaricomycotina; yet our clone library results indicate that only $\sim 13 \%$ of sequences amplified with ITS1F-LR21 are from fungal groups outside the Agaricomycotina. The assumption that each TRF represents a distinct species is also known not to be true (Edwards \& Turco 2005; Avis et al. 2006). In silico digests of the 139 OTU recovered in this study suggest that HaeIII digests were $\sim 90 \%$ effective in resolving rDNA ITS-28S genotypes defined at the $97 \%$ similarity level (Edwards, unpublished data). Moreover the pattern of TRF distribution between forest stands parallel that of ITS1F-LR21 OTU distribution (Fig. S2 Supporting information). Therefore, we believe that the TRFLP data presented here are a reasonable approximation of species distributions across this landscape. Given this, the comparatively low 


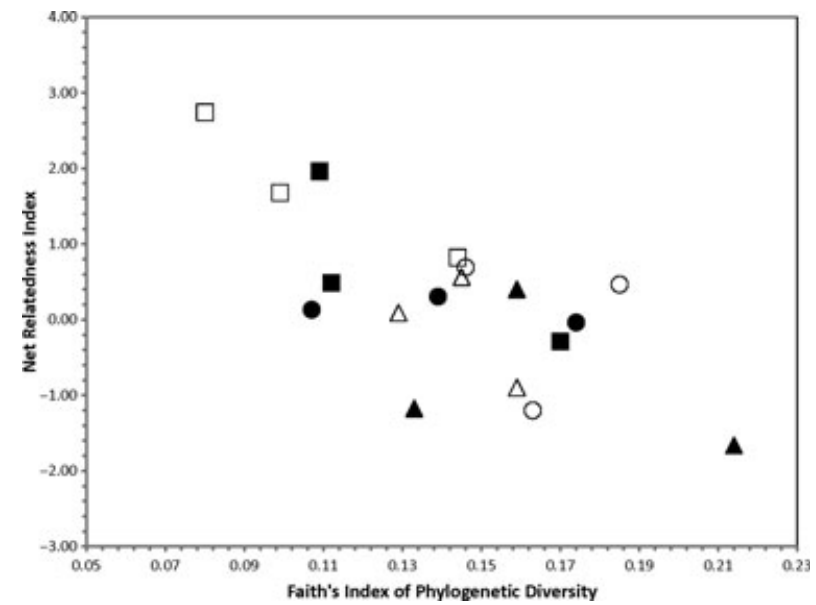

Fig. 4 Relationship between phylogenetic diversity and net relatedness index in the soil and forest floor Agaricomycotina communities of three temperate upland forests. Soil communities; open symbols. Forest floor communities; closed symbols. BOWO, squares; $\mathrm{SMRO}$, circles, SMBW, triangles.

explicatory value of ecosystem characteristics was more likely due to the high proportion of singletons in the data, even though their influence was down-weighted in our analysis. However, a recent study in which singletons were excluded from gradient analysis assigned a similarly low explicatory value to forest type (DeBellis et al. 2006). Forest characteristics clearly define some portion of the $\beta$-niche of many Agaricomycotina, although the distribution of most species is too patchy for strong ecological inference.

\section{Are the Agaricomycotina communities of temperate forest ecosystems phylogenetically distinct?}

In addition to being species rich, fungal communities are often phylogenetically diverse. In these ecosystems, 461 rDNA clones recovered 139 species from $>50$ genera in at least 15 orders of Agaricomycotina. In comparison, using similar approaches to cloning and species definitions, Porter et al. (2008) recovered species from 17 orders in a study of hemlock-dominated forests and Lynch \& Thorn (2006) found species from 11-12 orders in a study of grass and agricultural lands. Despite a combination of broad taxonomic range, limited representation at finer taxonomic scales (i.e. within families and genera) and limited resolution and support for phylogenetically defined clades (Supporting information Fig. S3), our results revealed significant differences between the forest floor and soil communities, and moreover, between the soil communities (Fig. 3).

UniFrac measures the distance between communities as the fraction of the total branch length of a phylogenetic tree that is unique to each environment; the assumption is that if membership of a community requires lineage - specific adaptations, then that community should share less branch length with other communities than if membership to all communities was open (Lozupone \& Knight 2005; Cavender-Bares et al. 2009). The significant UniFrac differences that we observed between forest floor and soil communities parallel the TRFLP results and reflect differential distribution of Agaricomycotina lineages at various taxonomic levels. Tremellomycetes generally were preferentially recovered from the forest floor, whereas within the Agaricomycetes, Sistotrema (Cantharellales), Mycena (Agaricales), and Clitocybe (Agaricales) were exclusive to forest floor. In contrast, Sebacinales, Cortinarius (Agaricales), Russula (Russulales), Thelephora (Thelephorales), Piloderma (Atheliales) Trechispora (Trechisporales), Collybia/Gymnopus (Agaricales) and the Clavariaceae (Agaricales) were exclusive to the soil horizon. To some extent, this stratification reflects a life strategy change from saprotrophic in the forest floor to mycorrhizal in the fine root rich soil horizon. However, the extent to which mycorrhizal species dominate community diversity in temperate soil horizons can differ considerably, and in this study ranged from $>90 \%$ to $<40 \%$ (Supplementary material Fig. 4). Preferential distributions of saprotrophic lineages such as the Clavariaceae also drive the clear phylogenetic differences between forest floor and soil communities. Although we found a significant difference between the forest floor and soil communities, we found no significant phylogenetic differences between the forest floor communities of the three ecosystems, although they did appear to differ slightly in phylogenetic diversity (Table 2, Fig. 4). Moreover, forest floor communities showed the greatest degree of species overlap (Fig. 2). Our results suggest that differences in forest floor biochemistry between temperate hardwood ecosystems (Table 1) exert a comparatively small influence on saprotrophic Agaricomycotina community composition and $\beta$-diversity.

In comparison with the forest floor communities, we observed greater differences between the Agaricomycotina communities of the soil horizon. Specifically, the community of BOWO, which was dominated by mycorrhizal species, differed significantly from the communities of both SMRO and SMBW. This difference is clearly driven by the phylogenetic differences between an ectomycorrhizal Cortinarius - Russula dominated community and less mycorrhizal dominated communities. We also observed a marginally significant phylogenetic difference between SMRO and SMBW, and this appears to reflect the differential distribution of ectomycorrhizal Quercus associated species and of putatively endomycorrhizal Sebacina species. 
Might temperate forest ecosystems act as habitat filters?

Despite evidence of species- and lineage-level sorting between the three ecosystems, generally the Agaricomycotina communities exhibited weak phylogenetic structure. Generally, local species assemblages can be seen as subsets of a larger species pool (Kraft et al. 2007). In comparison with this larger pool, the species in local assemblages may be more closely related than expected by chance (clustered), less closely related than expected by chance (overdispersed), or exhibit no phylogenetic structure (random). Interpretation of these patterns in terms of ecological process requires knowledge of the evolutionary history of life strategy/ecophysiological traits (Webb et al. 2002; Cavender-Bares et al. 2004; Kraft et al. 2007). For example, if different ecosystems favour different traits, and if these traits ( $\beta$-niche traits, sensu Silvertown et al. 2006) evolve conservatively, then communities should exhibit little phylogenetic similarity and strong phylogenetic clustering (Lozupone \& Knight 2005; Cavender-Bares et al. 2006; Kraft et al. 2007). Conversely, if ecological traits generally are conserved and habitat filtering is less important than limiting similarity, the communities of distinct ecosystems may have considerable phylogenetic overlap and even be phylogenetically overdispersed. However, if $\beta$-niche traits are convergent, strong habitat filtering should also lead to phylogenetic overlap and phylogenetic overdispersion, while strong limiting similarity leads to phylogenetically random or even possibly clustered patterns (Cavender-Bares et al. 2004; Kraft et al. 2007).

We observed significant phylogenetic structure only in the Agaricomycete - dominated forest floor and soil horizon communities of the BOWO ecosystem, in which phylogenetic clustering indicated that species in this community were more closely related than expected by chance (Table 3). While phylogenetic studies of microorganisms remain rare (Vamosi et al. 2009), bacterial communities have been found to be phylogenetically clustered in a range of environments, and this has been interpreted as evidence of habitat filtering, even though trait information is lacking (Horner-Devine \& Bohannan 2006; Bryant et al. 2008). Detailed trait information similar to that available for plants (Ackerly 2003) is lacking for fungi, and in the absence of such data, conclusions based on phylogenetic structure alone must be considered speculative. Mutualism is often a conserved generic trait and is believed to have multiple independent origins within the Agaricomycotina (Hibbett et al. 2000). Mutualism is clearly an important trait when considering successful colonization of the fine-root rich soil horizon in the BOWO ecosystem, in which mycorrhizal species accounted for $>90 \%$ of the community
(Table 1., Supplementary material), and evolutionary conservation of this life strategy within speciose genera, such as Russula and Cortinarius, largely explains the phylogenetic clustering of this community and suggests that this environment may act as a habitat filter. However, phylogenetic clustering was also observed in the Agaricomycete community colonizing the forest floor of the BOWO ecosystem; species from the saprotrophic Mycena and Clitocybe and the predominantly saprotrophic Sistotrema genera were found in this environment. Therefore, traits such as the ability to maintain growth during periods of low moisture availability may be more generally important to colonization of this ecosystem, and the degree to which such traits show evolutionary conservatism requires further study.

In contrast to the BOWO ecosystem, the Agaricomycotina communities of the two maple - dominated forest ecosystems were more phylogenetically diverse and exhibited no significant phylogenetic structure. Conceivably, this might reflect a high degree of convergent trait evolution and a community assembly process dominated by competitive interactions (Webb et al. 2002). More recently Kembel \& Hubbell (2006) and Kraft et al. (2007) have proposed that random phylogenetic patterns may also result when density dependent and environmental filtering processes are balanced or weak, or when neutral processes dominate community assembly. Dispersal limitation and high local variability in spore rain have been shown to affect fungal community composition (Vasiliauskas et al. 2005; Peay et al. 2007) and may lead to a lack of phylogenetic signal, especially if species have similar ecological traits or if trait evolution is random or convergent.

Lack of phylogenetic signal may also reflect methodological limitations (Swenson et al., 2009). In this study, neither spatial nor taxonomic scale exerted strong influence on the results, but both should probably be examined in more detail in future work. Although Unifrac found only a marginally significant difference in the SMRO and SMBW fungal communities, close inspection of the data reveals that, as described above, preferential and restricted distribution of some lineages (e.g. Cortinariaceae and Sebacinales) characterize the soil communities of these two ecosystems. Therefore, future family or genus level studies may yet reveal evidence of phylogenetic structure. Sampling intensity also needs to be addressed in future fungal community phylogenetic studies. Based on our estimates of species richness, we may have recovered between 36 to $62 \%$ of species from these sites, although these numbers have to be considered cautiously as they do not account for ribosomal copy number variation or PCR bias. It is likely that further sampling would reveal more species, and moreover that sampling efficiency was lower in the more 
diverse SMBW ecosystem. Finally, it is plausible that altering the scale of the reference community may lead to greater evidence of phylogenetic structure in the SMRO and SMBW ecosystems (Swenson et al., 2006; Cavender-Bares et al. 2006). Here, the reference community was defined solely by the samples, and within this context, the BOWO communities appear phylogenetically clustered. A more extensive reference community which integrated species information from other northern temperate forest ecosystems might also reveal phylogenetic structure in the SMRO and SMBW ecosystems.

\section{Conclusions}

Our results demonstrate that the distributions of Agaricomycotina species across a heterogeneous forested landscape are sensitive to the floristic and edaphic characteristics of distinct forest ecosystems. Habitat heterogeneity is therefore an important factor when considering fungal $\beta$-diversity. Moreover, our results provide a first indication of landscape-scale phylogenetic sorting, whereby the variation in community composition between ecosystems reflects the preferential distributions of distinct evolutionary lineages within the Agaricomycotina. Phylogenetic sorting was especially clear between the forest floor and soil communities, where it was in part the result of an ecological split between saprotrophic and mycorrhizal communities. Phylogenetic sorting was also observed between communities colonizing the soil horizon of the three ecosystems, and appeared to reflect differences in host plant specialization between mycorrhizal groups such as Cortinarius and Sebacina. There was no evidence of phylogenetic sorting in the forest floor communities. Habitat heterogeneity therefore appears to be an important factor when considering fungal 'phylo'- $\beta$-diversity (sensu Graham \& Fine 2008).

Although we found evidence for phylogenetic sorting between forest floor and soil, and between soils, significant phylogenetic clustering was only observed in one of the three ecosystems that we examined, and generally phylogenetic structure was inversely correlated with phylogenetic diversity. As such, our results suggest that within at least some lineages in the Agaricomycotina, phylogenetic relatedness may be an indication of ecological similarity, at least at the $\beta$-niche scale. Moreover our results suggest that some forest ecosystems may act as habitat filters. Further progress in understanding the determinants of Agaricomycotina $\beta$-diversity and community assembly process requires greater understanding of the correlation between ecological similarity and phylogenetic relatedness (Losos 2008).

\section{References}

Ackerly DD (2003) Community assembly, niche conservatism, and adaptive evolution in changing environments. International Journal of Plant Science, 164, S165-S184.

Albert DA, Denton SR, Barnes BV (1986) Regional Landscape Ecosystems of Michigan, School of Natural Resources, University of Michigan, Ann Arbor.

Avis PG, Dickie IA, Mueller GM (2006) A 'dirty' business: testing the limitations of terminal restriction fragment length polymorphism (TRFLP) analysis of soil fungi. Molecular Ecology, 15, 873-882.

Bååth E (1988) A critical examination of the soil washing technique with special reference to the effect of the size of the soil particles. Canadian Journal of Botany, 66, 1566-1569.

Barnes BV, Pregitzer KS, Spies TA, Spooner VH (1982) Ecological Forest site classification. Journal of Forestry, 80, 493-498.

Blackwood CB, Waldrop MP, Zak DR, Sinsabaugh RL (2007) Molecular analysis of fungal communities and laccase genes in decomposing litter reveals differences among forest types but no impact of nitrogen deposition. Environmental Microbiology, 9, 1306-1316.

Bruns TD, Bidartondo MI, Taylor DL (2002) Host specificity in ectomycorrhizal communities: what do the exceptions tell us? Integrative and Comparative Biology, 42, 352-359.

Bryant JA, Lamanna C, Morlon H, Kerkhoff AJ, Enquist BJ, Green JL (2008) Microbes on mountainsides: contrasting elevational patterns of bacterial and plant diversity. PNAS, 105, 11505-11511.

Cavender-Bares J, Ackerly DD, Baum DA, Bazzaz FA (2004) Phylogenetic overdispersion in Floridian Oak Communities. The American Naturalist, 163, 823-843.

Cavender-Bares J, Keen A, Miles B (2006) Phylogenetic structure of Floridian plant communities depends on taxonomic and spatial scale. Ecology, 87, S109-S122.

Cavender-Bares J, Kozak KH, Fine PVA, Kembel SW (2009) The merging of community ecology and phylogenetic biology. Ecology Letters, 12, 693-715.

DeBellis T, Kernaghan G, Bradley R, Widden P (2006) Relationships between stand composition and ectomycorrhizal community structure in boreal mixed-wood forests. Microbial Ecology, 52, 114-126.

Dickie IA, FitzJohn RG (2007) Using terminal restriction fragment length polymorphism (T-RFLP) to identify mycorrhizal fungi: a methods review. Mycorrhiza, 17, 259-270.

Drummond AJ, Ashton B, Cheung M et al. (2008) Geneious v.8.1. http://www.geneious.com/.

Edwards IP, Turco RF (2005) Inter- and intraspecific resolution of rDNA TRFLP assessed by computer-simulated restriction analysis of a diverse collection of ectomycorrhizal fungi. Mycological Research, 109, 212-226.

Egert M, Friedrich MW (2003) Formation of pseudo-terminal restriction fragments, a PCR-related bias affecting terminal restriction fragment length polymorphism analysis of microbial community structure. Applied and Environmental Microbiology, 69, 2555-2562.

Faith DP (1992) Conservation estimation and phylogenetic diversity. Biological Conservation, 61, 1-10.

Gardes M, Bruns TD (1993) ITS primers with enhanced specificity for basidiomycetes - application to the 
identification of mycorrhizas and rusts. Molecular Ecology, 2, 113-118.

Graham CH, Fine PVA (2008) Phylogenetic beta diversity:" linking ecological and evolutionary processes across space in time. Ecology Letters, 11, 1265-1277.

Hawksworth DL (2001) The magnitude of fungal diversity: the 1.5 million species estimate revisited. Mycological Research, $105,1422-1432$.

Hibbett DS (2006) A phylogenetic overview of the Agaricomycotina. Mycologia, 98, 917-925.

Hibbett DS, Gilbert L-B, Donoghue MJ (2000) Evolutionary instability of ectomycorrhizal symbioses in basidiomycetes. Nature, 407, 506-508.

Hopple JS, Vilgalys R (1994) Phylogenetic relationships among coprinoid taxa and allies based on data from restriction site mapping of nuclear rDNA. Mycologia, 86, 96-107.

Horner-Devine MCB, Bohannan JM (2006) Phylogenetic clustering and overdispersion in bacterial communities. Ecology, 87, S100-S108.

Host GE, Pregitzer KS, Ramm CW, Lusch DP, Cleland DT (1988) Variation in overstory biomass among glacial landforms and ecological land units in northwestern Lower Michigan. Canadian Journal of Forest Research, 18, 659-668.

Hughes JB, Hellmann JJ, Rickets TH, Bohannan BJ (2001) Counting the uncountable: statistical approaches to estimating microbial diversity. Applied and Environmental Microbiology, 67, 4399-4406.

Kembel SW, Hubbell SP (2006) The phylogenetic structure of a Neotropical forest tree community. Ecology, 87, S86-S99.

Kerr J, Southwood TRE, Cihlar J (2001) Remotely sensed habitat diversity predicts butterfly species richness and community similarity in Canada. PNAS, 98, 11365-11370.

Kraft NJB, Cornwell WK, Webb CO, Ackerly DD (2007) Trait evolution, community assembly, and the phylogenetic structure of ecological communities. The American Naturalist, 170, 271-283.

Kranabetter JM, Durall DM, MacKenzie WH (2009) Diversity and species distribution of ectomycorrhizal fungi along productivity gradients of a southern boreal forest. Mycorrhiza, 19, 99-111.

Laliberté E, Paquette A, Legendre P, Bouchard A (2009) Assessing the scale-dependent importance of niches and other spatial processes on beta diversity: a case study from a temperate forest. Oecologia, 159, 377-388.

Lindahl BD, Ihrmark K, Boberg J et al. (2007) Spatial separation of litter decomposition and mycorrhizal nitrogen uptake in a boreal forest. New Phytologist, 173, 611-620.

Losos JB (2008) Phylogenetic niche conservatism, phylogenetic signal and the relationship between phylogenetic relatedness and ecological similarity between species. Ecology Letters, 11, 995-1007.

Lozupone C, Knight R (2005) UniFrac: a new phylogenetic method for comparing microbial communities. Applied and Environmental Microbiology, 71, 8228-8235.

Lynch MDJ, Thorn RG (2006) Diversity of basidiomycetes in Michigan agricultural soils. Applied and Environmental Microbiology, 72, 7050-7056.

Molina R, Massicote H, Trappe JM (1992) Specificity phenomena in mycorrhizal symbiosis: community-ecological consequences and practical implications. In: Mycorrhizal
Functioning: An Integrative Plant-Fungal Process (ed Allen MF). pp. 357-423. Wiley, New York, NY, USA.

Nantel P, Neumann P (1992) Ecology of ectomycorrhizalbasidiomycete communities on a local vegetation gradient. Ecology, 73, 99-117.

Nilsson RH, Kristiansson E, Ryberg M, Hallenberg N, Larsson K-H (2008) Intraspecific ITS variability in the Kingdom Fungi as expressed in the international sequence databases and its implication for molecular species identification. Evolutionary Bioinformatics, 4, 193-201.

O'Brien HE, Parrent JL, Jackson JA, Moncalvo J-M, Vilgalys R (2005) Fungal community analysis by large-scale sequencing of environmental samples. Applied and Environmental Microbiology, 71, 5544-5550.

Peay KG, Bruns TD, Kennedy PG, Bergemann SE, Garbelotto M (2007) A strong species-area relationship for eukaryotic soil microbes: island size matters for ectomycorrhizal fungi. Ecology Letters, 10, 470-480.

Pickett STA, Bazzaz FA (1978) Organization of an assemblage of early successional species on a soil moisture gradient. Ecology, 59, 1248-1255.

Porter TM, Skillman JE, Moncalvo JM (2008) Fruiting body and soil rDNA sampling detects complimentary assemblage of Agaricomycotina (Basidiomycota, Fungi) in a hemlockdominated forest plot in southern Ontario. Molecular Ecology, 17, 3037-3050.

Posada D, Crandall KA (1998) MODELTEST: testing the model of DNA substitution. Bioinformatics, 14, 817-818.

Schloss PD, Handelsman J (2005) DOTUR, a computer program for defining operational taxonomic units and estimating species richness. Applied and Environmental Microbiology, 71, 1501-1506.

Silvertown J, Dodd M, Gowing D, Lawson C, McConway K (2006) Phylogeny and the hierarchical organization of plant diversity. Ecology, 87, S39-S49.

Smith SE, Read DJ (1997) Mycorrhiza Symbiosis, 2nd edn. , Harcourt Brace Co., Publishers Academic Press, London.

Swenson NG (2009) Phylogenetic Resolution and Quantifying the Phylogenetic diversity and dispersion of communities. PLOS ONE, 4, e4390.

Tamura K, Dudley J, Nei M, Kumar S (2007) MEGA4: Molecular Evolutionary Genetics Analysis (MEGA) software version 4.0. Molecular Biology and Evolution, 24, 1596-1599.

Taylor JW, Jacobsen DJ, Kroken S et al. (2000) Phylogenetic species recognition and species concepts in fungi. Fungal Genetics and Biology, 31, 21-32.

Ter Braak CJF (1986) Canonical Correspondence Analysis: a new eigenvector technique for multivariate direct gradient analysis. Ecology, 67, 1167-1179.

Thompson JD, Gibson TJ, Plweniak F, Jenamougin F, Higgin DG (1997) The CLUSTAL_ $X$ windows interface: flexible strategies for multiple sequence alignment aided by quality analysis tools. Nucleic Acids Research, 25, 4876-4882.

Tyler G (1992) Tree species affinity of decomposer and ectomycorrhizal macrofungi in beech (Fagus sylvatica L.), oak (Quercus robur L.) and hornbeam (Carpinus betulus L.) forests. Forest Ecology and Management, 47, 269-284.

Vamosi SM, Heard SB, Vamosi C, Webb CO (2009) Emerging patterns in the comparative analysis of phylogenetic community structure. Molecular Ecology, 18, 572-592. 
Vasiliauskas R, Lygis V, Larsson K-H, Stenlid J (2005) Airborne fungal colonization of coarse woodly debris in North Temperate Picea abies forest: impact of season and local spatial scale. Mycological Research, 109, 487-496.

Villeneuve N, Grandtner MM, Fortin JA (1989) Frequency and diversity of ectomycorrhizal and saprophytic macrofungi in the Laurentide Mountains of Quebec. Canadian Journal of Botany, 67, 2616-2629.

Webb CO (2000) Exploring the phylogenetic structure of ecological communities: an example for rain forest trees. American Naturalist, 156, 145-155.

Webb CO, Ackerly DD, McPeek MA, Donoghue MJ (2002) Phylogenies and community ecology. Annual Review of Ecology and Systematics, 33, 475-505.

Whittaker RH (1972) Evolution and measurement of species diversity. Taxon, 21, 213-251.

Zak DR, Host GE, Pregitzer KS (1990) Regional variability in nitrogen mineralization, nitrification, and overstory biomass in northern Lower Michigan. Canadian Journal of Forest Research, 19, 1521-1526.

Ivan Edwards is a microbial community ecologist. His research focuses are the factors that affect the distributions of fungal species and the response of fungal communities during environmental change. He is Senior Research Fellow at the University of Michigan. Donald Zak is an ecosystem ecologist with broad interests in the connections between microbial community composition and function, and the implications of these connections for the biogeochemical cycling of carbon and nitrogen in soil. He is Collegiate Professor at the University of Michigan.

\section{Supporting Information}

Additional supporting information may be found in the online version of this article.
Table S1 rDNA ITS-28S genotypes from upland temperate forest ecosystems

Fig. S1 Collectors curves. Top: Chao 1; Bottom: Jackknife. ITS - derived curves are indicated by solid symbols, and $28 \mathrm{~S}$ curves by open symbols.

Fig. S2 Comparison of the distribution of TRFP and clone library Operational Taxonomic Units across nine temperate upland forest stands. Top: forest floor communities; Bottom: soil communities.

Fig. S3 Phylogenetic placement (Neighbour-joining analysis with Kimura 2-parameter model of partial rDNA 28S sequences) of 139 Agaricomycotina operational taxonomic units (OTU) recovered from rDNA random clone libraries of the forest floor and soil of three contrasting upland forest ecosystems. Source of OTU (forest floor or soil) is indicated, and best BLAST match is listed in parentheses. Morchella esculenta and Morchella elata were used as the outgroup.

Fig. S4 Functional guild breakdown of basidiomycete rDNA ITS-28S operational taxonomic units in three temperate upland forest ecosystems in upper lower Michigan, USA. Data are expressed as percentage of OTU in the clone libraries representing each system. BOWO, black oak - white oak/Vaccinium, SMRO, sugar maple- red oak/Maianthemum, SMBW, Sugar maple basswood/Osmorhiza. Solid black, saprotrophs; hashed, ectomycorrhizal; grey, unclassified.

Please note: Wiley-Blackwell are not responsible for the content or functionality of any supporting information supplied by the authors. Any queries (other than missing material) should be directed to the corresponding author for the article. 